\title{
IMPACTRIMONIO: TECNOLOGÍAS DE LA INFORMACIÓN APLICADAS A LA VALORACIÓN DEL IMPACTO ECONÓMICO DE LA CULTURA
}

\section{Pau Rausell-Köster, Vicente Coll-Serrano, Raúl Abeledo-Sanchis y Francisco Marco-Serrano}

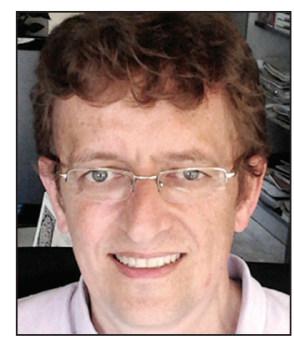

Pau Rausell-Köster es profesor del Departamento de Economía Aplicada de la Universitat de València y miembro del Instituto Interuniversitario para el Desarrollo Local. Desde 1996 dirige la Unidad de investigación en Economía de la Cultura y Turismo (Econcult). http://orcid.org/0000-0003-2274-7423

Instituto de Desarrollo Local, Universidad de Valencia Avda. dels Tarongers, s/n. 46021 Valencia, España pau.rausell@uv.es

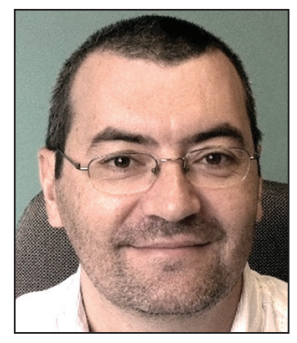

Vicente Coll-Serrano es profesor del Departamento de Economía Aplicada de la Universitat de València y miembro del grupo de investigación MC2 (Métodos Cuantitativos para la Medición de la Cultura). Ha participado en proyectos de transferencia de diseño e implementación de sistemas de indicadores culturales para organismos y administraciones públicas.

http://orcid.org/0000-0002-7813-7379

Universidad de Valencia, Departamento de Economía Aplicada Avda. dels Tarongers, s/n. 46021 Valencia, España vicente.coll@uv.es

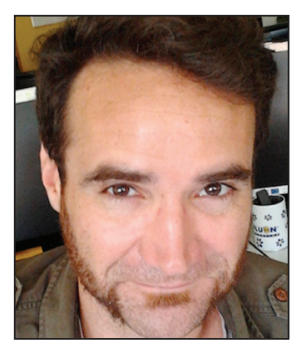

Raúl Abeledo-Sanchis es profesor asociado del Departamento de Economía Aplicada de la Universitat de València y miembro de la Unidad de Investigación en Economía de la Cultura y Turismo (Econcult). Ha participado en proyectos de cultura y desarrollo, como el proyecto europeo Sostenuto de creatividad, cultura e innovación económica y social.

http://orcid.org/0000-0002-5762-1874

Universidad de Valencia, Departamento de Economía Aplicada Avda. dels Tarongers, s/n. 46021 Valencia, España rabeledo@gmail.com

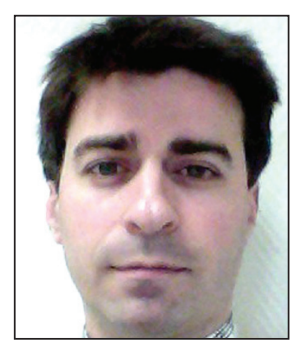

Francisco Marco-Serrano es profesor del Departamento de Economía de GSM London (Reino Unido) y colaborador externo del grupo de investigación Econcult de la Universitat de València. Ha participado en proyectos de investigación y es autor de libros, artículos e informes profesionales sobre el impacto y la dimensión económica de la cultura.

http://orcid.org/0000-0003-1806-2624

K/P/K - Key Productivity Konsulting y Department of Economics at GSM London Meridian House, Royal Hill, Greenwich, London SE10 8RD, United Kingdom fmarco@kpkonline.com

\section{Resumen}

Se propone un servicio de información, Impactrimonio, que evalúa el impacto económico que un museo, biblioteca, festival o elemento patrimonial genera sobre el territorio. A partir de la información contenida en las tablas input-output y los datos proporcionados por el propio usuario a través de cuestionarios, Impactrimonio estima los efectos directos, indirectos e inducidos para cuantificar el impacto. Se describen los principales elementos que configuran la arquitectura y la interfaz del sistema.

\section{Palabras clave}

Impacto económico, Servicio informacional, Efectos económicos, Actividad cultural, Gestión cultural, Tecnologías de la información. 


\section{Title: Impactrimonio: information technologies applied to assessing the economic impact of culture}

\section{Abstract}

This paper proposes an informational service, Impactrimonio, to assess the economic impact of a particular museum, library, festival or heritage element on the economic development of a particular territory. This service takes on increasing importance in the context of the new development paradigm linked to the Cultural Economy. From the information contained in the input-output tables and the data supplied by the user on various questionnaires, Impactrimonio estimates the direct, indirect and induced effects necessary to quantify the economic impact of the cultural activity.

\section{Keywords}

Economic impact, Informational service, Economic effects, Cultural activity, Cultural management, Information technologies.

Rausell-Köster, Pau; Coll-Serrano, Vicente; Abeledo-Sanchis, Raúl; Marco-Serrano, Francisco (2013). “Impactrimonio: tecnologías de la información aplicadas a la valoración del impacto económico de la cultura". El profesional de la información, julio-agosto, v. 22, n. 4, pp. 309-314.

http://dx.doi.org/10.3145/epi.2013.jul.06

\section{Introducción: economía de la información e Impactrimonio}

El concepto de economía de la información tiene una interpretación polisémica. Puede entenderse como:

- aportación de la microeconomía, que destaca que los agentes económicos no tienen conocimientos perfectos sobre los mercados ni sobre el nivel tecnológico y, en consecuencia, los costos de información distorsionan de manera relevante el funcionamiento de los equilibrios previstos por la teoría tradicional (Stigliz, 2000) ${ }^{1}$;

- estructura económica del sector de la información, formado por los servicios e industrias de la información y contenidos digitales, pero también telecomunicación, electrónica, videojuegos, y otras actividades TIC (desde electrónica del automóvil al mantenimiento y comercialización de equipos electrónicos y componentes);

- relación con el análisis de un "bien", denominado información, que se comporta de una determinada manera. Es la aproximación de autores como Shapiro y Varian (1999), quienes consideran "información" todo aquello que se puede digitalizar y sobre lo que existen demandas potenciales expresables en términos monetarios.

Este artículo se plantea en el último contexto. Se propone un servicio informacional que a través de la automatización de algoritmos calcula el impacto económico que un evento, museo o elemento patrimonial genera sobre el territorio. El "valor del servicio informacional" propuesto es creciente, ya que aunque podemos constatar que la dimensión simbólica de una comunidad siempre ha tenido un papel relevante en la conformación del espacio socioeconómico, esta influencia se ha ido reforzando en las dos últimas décadas (Unctad, 2010; Rausell-Köster et al., 2007; 2012).

Como señalan diversas formulaciones sobre la sociedad del conocimiento, el capitalismo cognitivo o la sociedad de la información, y tal como indica la propia Comisión Europea (2010), las fábricas están siendo remplazadas por comunidades creativas cuya materia prima es su habilidad para imaginar, crear e innovar. Así, desde principios del siglo XXI se están haciendo esfuerzos para aportar datos cuantitativos que delimiten la dimensión económica de las actividades culturales y las industrias creativas. Una de las investigaciones realizadas en esta línea ha demostrado que el sector de las industrias culturales y creativas en Europa representa el 2,6\% del PIB, genera más de 5 millones de empleos y es uno de los sectores más dinámicos, con altos índices de crecimiento (KEA, 2006). La cultura amplía la frontera de posibilidades de nuestro futuro. En este momento sería una irresponsabilidad por parte de Europa no aprovechar con inteligencia esta circunstancia.

\section{Las fábricas están siendo reemplazadas por comunidades creativas cuya mate- ria prima es su habilidad para imaginar, crear e innovar}

En efecto, a esta centralidad de la cultura para el desarrollo local y regional (UCLG, 2004) se le suma la actual situación de crisis económica: las restricciones de los presupuestos públicos y privados refuerzan aún más la necesidad de estrategias de medición y herramientas de análisis que justifiquen el impacto económico y el valor social de las iniciativas culturales, tal y como demanda la propia Comisión Europea (2010).

En este marco se ha diseñado Impactrimonio, una herramienta basada en una interface web que calcula el impacto económico de un museo, un festival o un emplazamiento patrimonial, a partir de las informaciones aportadas por los usuarios y en combinación con la información sobre los flujos económicos del territorio.

\section{Arquitectura de Impactrimonio}

Es una herramienta implementada en una plataforma web, que facilita al usuario la estimación del impacto económico derivado de una determinada actividad cultural. Está configurada para realizar la evaluación de tres tipos de actividad: 


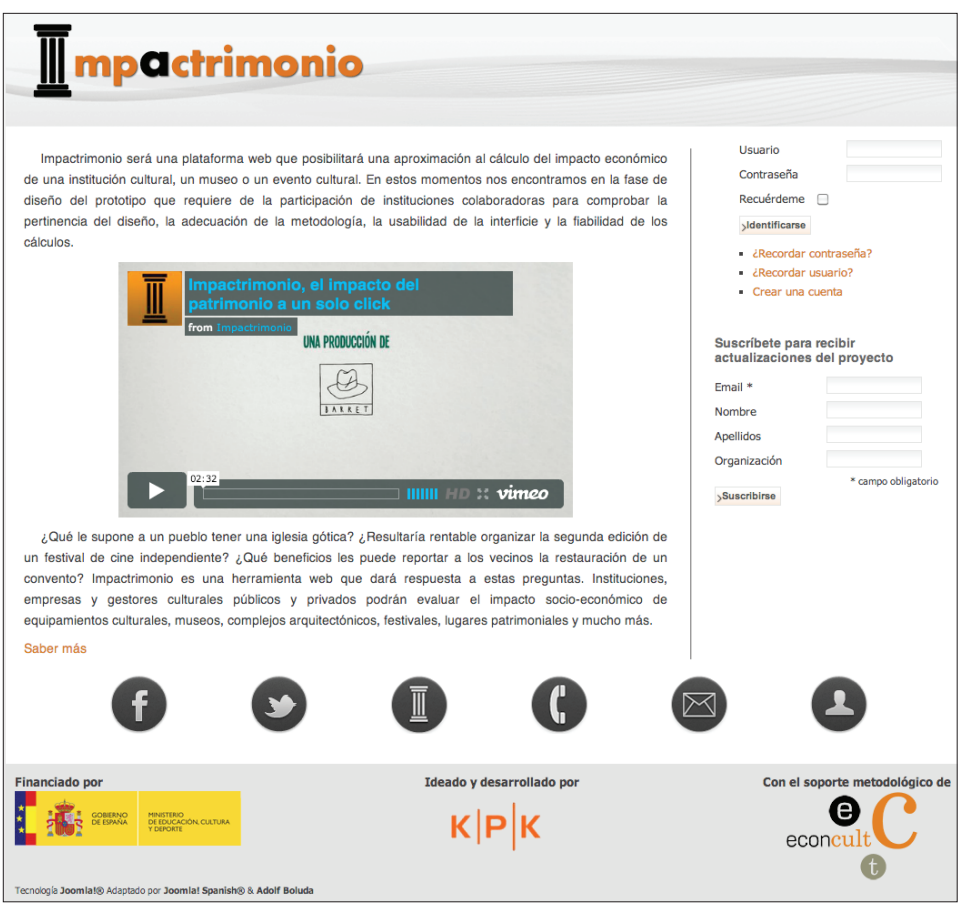

Figura 1. Página inicial nomía permite evaluar cómo afecta el gasto de un sector en el resto, es decir las TIO permiten recoger los flujos de bienes y servicios entre los sectores de la economía a nivel desagregado por ramas de actividad. Supongamos que un sector adquiere materiales de otros sectores proveedores para poder realizar su actividad económica; a su vez éstos recurrirán a sus respectivos proveedores para obtener las materias primas para su producción. Estos efectos se transmiten hacia adelante (clientes) y hacia atrás (proveedores) en un movimiento de transmisión y difusión que se expande a lo largo de la cadena de valor, y que viene caracterizado por la estructura de la TIO.

Los cálculos matemáticos necesarios para la estimación de los efectos directos, indirectos e inducidos (Pulido; Fontela, 1993; Seeman, 2003) se han programado utilizando el lenguaje de programación $\mathrm{R}$, lenguaje de código abierto utilizado para cálculos estadísticos y análisis de datos. Se ha utilizado la integración $\mathrm{R}$ en web rApache, un entorno para servidor web Apache que permite ejecutar scripts en lenguaje $\mathrm{R}$ desde la web.
- museos;

- festivales;

- elementos del patrimonio construido.

Impactrimonio está basado en software libre y código propio. La base de la aplicación es Joomla, un gestor de contenidos que constituye la interfaz del usuario, sobre el que se han realizado modificaciones.

\section{Recopilación de datos y tratamiento de la información}

La información que maneja Impactrimonio procede de diversas fuentes. Por un lado se alimenta de la base de datos CulturaBase del Ministerio de Cultura, y de las tablas inputoutput (TIO) de España que, de acuerdo con el directorio del Ministerio de Educación, Cultura y Deportes, dispone de información de más de 1.500 museos, más de 16.000 elementos patrimoniales protegidos y un número creciente de festivales de distintas dimensiones de música, artes escénicas o del sector audiovisual. Por otro lado Impactrimonio gestiona la información suministrada por la propia institución.

Para capturar los datos de la institución se ha utilizado Limesurvey, una aplicación de código abierto para encuestas. Los diferentes cuestionarios diseñados en la aplicación se integran dentro de un iframe en Joomla, de forma que resulta transparente para el usuario y no tiene la sensación de que está utilizando otra herramienta.

La metodología empleada para la estimación de los efectos del ente o acto cultural (directos, indirectos e inducidos -ver explicación más abajo-) a considerar para determinar el impacto económico se apoya en el uso de las tablas input-output (Pulido; Fontela, 1993; Herrero-Prieto, 2002; Seeman, 2003; Devesa, 2006; Herrero-Prieto et al., 2006). Una TIO forma parte del marco de la contabilidad nacional y cuantifica -a nivel de oferta, demanda y rentas- las relaciones entre los sectores de la economía. Esta representación de la eco-

\section{Alimentación de datos y administración}

Para realizar el informe de impacto (ver apartado 3) se hace uso tanto de los datos introducidos por el usuario como de los procedentes de las TIO que se utilizan en cada caso, que depende de la localización geográfica de la institución a analizar y de su ámbito de impacto. Dispondremos de una TIO a nivel nacional y una por cada comunidad autónoma. Los datos se obtienen del Instituto Nacional de Estadística (INE). En cada caso son tablas de diferente tamaño y con datos divididos en varios sectores. Los sectores se han seleccionado según su importancia e interés para el estudio de impacto económico y cada una de las tablas se estandariza de forma que los cálculos se realizan siempre sobre tablas del mismo tamaño y sobre los mismos sectores.

La gestión de toda la información necesaria para realizar los oportunos cálculos estadísticos, así como la gestión de los usuarios, se realiza en la parte de administración de Joomla mediante la programación de un componente.

\section{Interfaz de Impactrimonio}

Está implementado en una plataforma web. En la página inicial, de libre acceso (figura 1), se proporciona información general sobre la aplicación así como información de contacto del blog y de perfiles en redes sociales (Facebook y Twitter).

\section{http://www.impactrimonio.es}

Para acceder al área restringida, en la que se puede obtener el informe del impacto económico de una actividad cultural o institución, el usuario debe realizar una solicitud de alta. Una vez aceptada, se le facilita un identificador de usuario y una contraseña. Esquemáticamente la lógica del proceso de funcionamiento es la que se muestra en la figura 2.

Para generar el informe de impacto económico se guía al usuario a través de formularios (figura 3) que deben ser 
cumplimentados para recopilar la información necesaria para realizar las estimaciones. Estos formularios se refieren a:

- perfil del usuario/a: datos del mismo y de la institución a la que está vinculado, indicando -entre otros- tipo de institución (museo, patrimonio construido o festival), área geográfica de impacto y el sector al que pertenece (público o privado);

- desglose de gastos: estructura de gastos de la institución de los últimos 5 años;

- desglose de ingresos: estructura de ingresos de la institución de los últimos 5 años;

- personal trabajador: información sobre el personal de la institución de los últimos 5 años.

- datos de visitantes y distribución del gasto: información sobre los visitantes y la distribución del gasto que realizan. Para obtener esta información, se facilita un modelo de cuestionario para que la institución capte la información necesaria entre sus visitantes.

A modo de ejemplo, en el formulario relativo al desglose de ingresos (figura 4) se solicita la aportación de datos económicos que se corresponden con los capítulos 3 a 9 del presupuesto de ingresos de las entidades sin fines de lucro. Concretamente, estos datos hacen referencia, sin ser exhaustivos, a:

- ingresos exigidos por la entidad en concepto de prestación de servicios y actividades realizadas (por ejemplo, cuotas por matriculaciones en cursos organizados, tasas por la emisión de certificados, etc.); los ingresos procedentes de la venta de entradas y los derivados de la externalización determinados servicios o los provenientes de venta de bienes relacionados con la actividad de la entidad como puede ser la venta de libros, revistas, dvds, etc.;

- transferencias corrientes recibidas de administraciones públicas o ingresos provenientes de donaciones, mecenazgo o patrocinio, tanto de entidades privadas como de familias y entidades sin ánimo de lucro;

- intereses de títulos y valores;

- ingresos derivados de la venta de inversiones reales;

- transferencias de capital recibidas;

- ingresos procedentes de la venta de activos financieros así como de aquellos procedentes del reintegro de préstamos concedidos y reintegros de depósitos y fianzas constituidas;

- ingresos procedentes de la emisión de deuda y la obtención de préstamos.

Junto con toda la información introducida por el usuario, para elaborar el informe técnico de impacto económico es necesario acceder a los datos contenidos en la TIO correspondiente, en función de la localización geográfica y del sector de la institución a analizar. Para ello se dispone de la TIO a nivel nacional y de las TIO de las comunidades autónomas, como se dijo, obtenidas del INE. Dado que en origen las TIO no eran homogéneas, fue necesario proceder a su estandarización, para que los posteriores cálculos se realizasen sobre tablas de la misma dimensión o tamaño y sobre los mismos sectores de actividad (seleccionados atendiendo a su importancia e interés para el estudio del impacto económico de la actividad o institución cultural). A nivel técnico, para la estandarización de las TIO se han programado unos diccionarios que traducen cada una a los sectores de la aplicación.

\section{La estimación de los efectos (directos, indirectos e inducidos) a considerar para determinar el impacto económico se basa en TIO}

La medición de la rentabilidad de un fenómeno cultural, al igual que sucede con los grandes eventos de ocio y las grandes infraestructuras, pasa por considerar su impacto económico total, ya que los efectos trascienden el ámbito físico de la empresa o entidad que lo promueve. Para determinar el impacto económico a partir de la información proporcionada por los usuarios (es necesario que éstos hayan cumplimentado todos los cuestionarios solicitados) y tomando como base metodológica las TIO, la aplicación Impactrimonio estima, como parte integrante del informe final, los siguientes efectos:

- Directos. Se corresponden con los gastos corrientes y dotacionales realizados por la actividad o institución cultural en diferentes conceptos (salarios, compras, alquileres, ejecución de programas, reformas, ampliaciones y adecuaciones, etc.) en el área geográfica de referencia y en un período de tiempo determinado. Además deben incluirse los gastos que realizan los visitantes como consecuencia del consumo del producto cultural (alojamiento, restauración, transporte, compras, entradas, etc.).

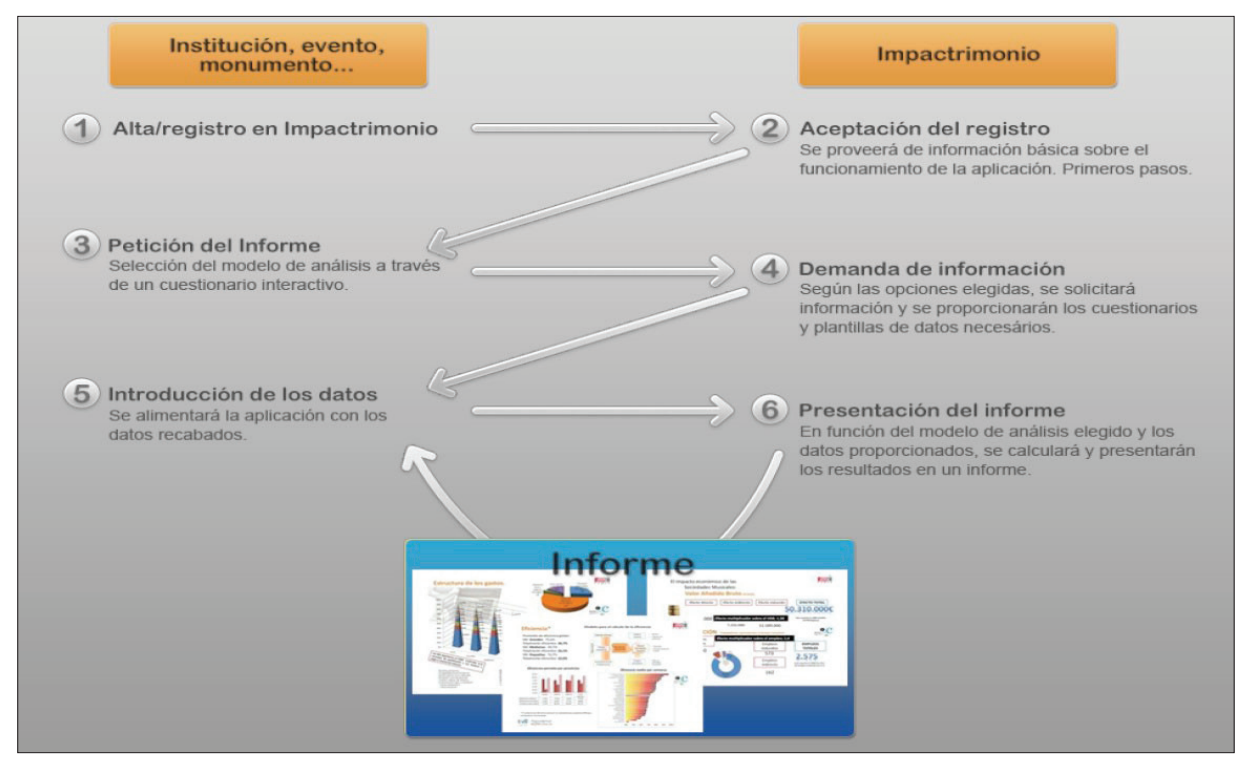

Figura 2. Proceso de funcionamiento de Impactrimonio 


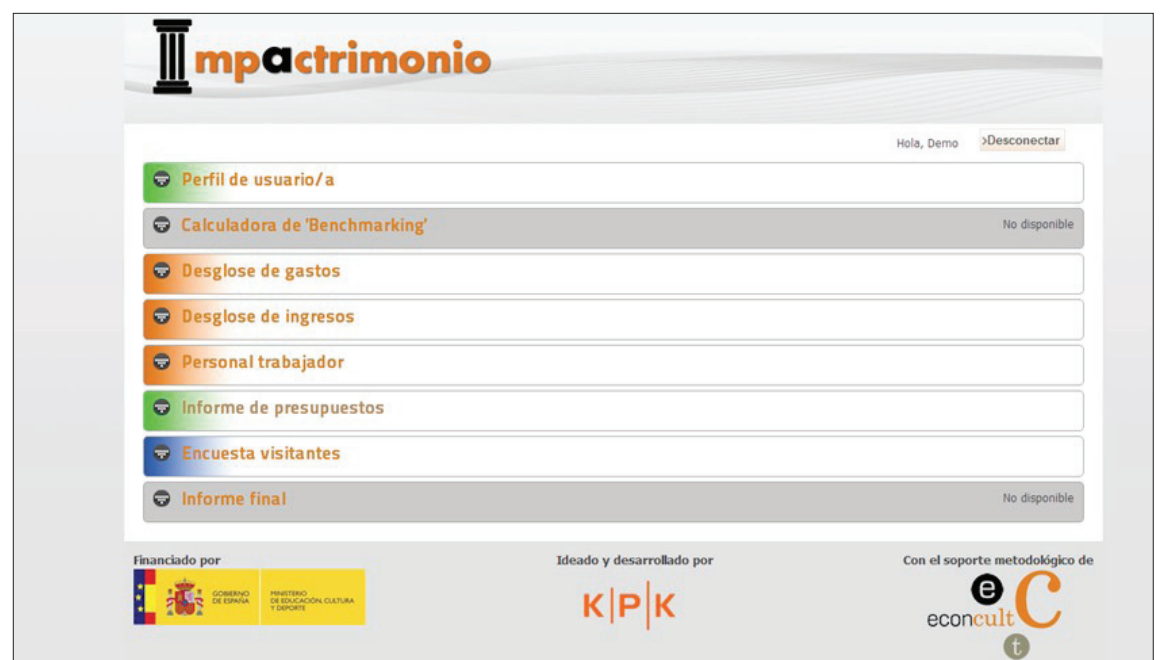

Figura 3. Formularios

- Indirectos. Son los derivados de la estructura reticular de las economías. Como consecuencia de una mayor venta, los proveedores de la institución cultural y/o de los establecimientos de restauración, hostelería, entre otros, incrementan a su vez el nivel de compras a sus respectivos proveedores, provocando un efecto dominó a nivel de la economía local / regional / nacional y/o, en su caso, internacional mediante importaciones.

- Inducidos. Son los efectos de renta, por ejemplo derivados del gasto en personal de las empresas, que inducen a un mayor consumo, produciéndose una activación económica que al tiempo genera efectos agregados dentro de la cadena de suministro.

El impacto total de una determinada actividad cultural se obtiene de la suma de los efectos directos, indirectos e inducidos. Además, su análisis permite evaluar la importancia relativa de una institución cultural mediante la estimación de su impacto económico en términos de valor añadido bruto, producción y empleo; así como sus repercusiones fiscales derivadas, a modo de ejemplo, de una mayor recaudación por IVA en el consumo o de IRPF por las nóminas.

Combinando toda la información disponible, el informe global se compone de:

- análisis de la estructura de los presupuestos de la institución comparada con la media de otras instituciones similares cuya información esté capturada en el sistema;

- informe de los visitantes comparados con las medias de los de otras instituciones similares que se encuentren en el sistema;

- estudio de impacto propiamente dicho que ofrece tanto los valores absolutos de los efectos económicos considerados, como los multiplicadores en términos de producción, valor añadido, empleo y efecto fiscal.
Impactrimonio se beneficia de cierto "efecto red", ya que a medida que el uso sea más extendido, las comparaciones con las medias de las instituciones capturadas será más precisa y ajustada, con lo que en términos analíticos aportará mucho más valor para la institución participante.

En definitiva el sistema combina dos fuentes de información estructurada: la de la propia organización en comparación con las similares, y la información sobre las relaciones económicas del territorio que queda recogida a través de las TIO. De esta combinación deriva el análisis del impacto económico que trata de detectar la contribución del evento o la institución en la transformación y generación de nuevos flujos de valor en las relaciones económicas.

\section{Consideraciones finales}

Impactrimonio se ha concebido como un instrumento flexible de análisis técnico que utiliza una metodología de estimación consolidada para evaluar el impacto económico, en una primera fase, de la actividad de museos, bibliotecas, festivales y elementos del patrimonio construido. En estos momentos puede darse por concluida la fase de prototipo, implementada en una plataforma web, para la que se ha contado con la participación de una veintena de instituciones colaboradoras (betatesters) a fin de comprobar la pertinencia del diseño, la adecuación de la metodología, la fiabilidad de los cálculos y la usabilidad de la interfaz.

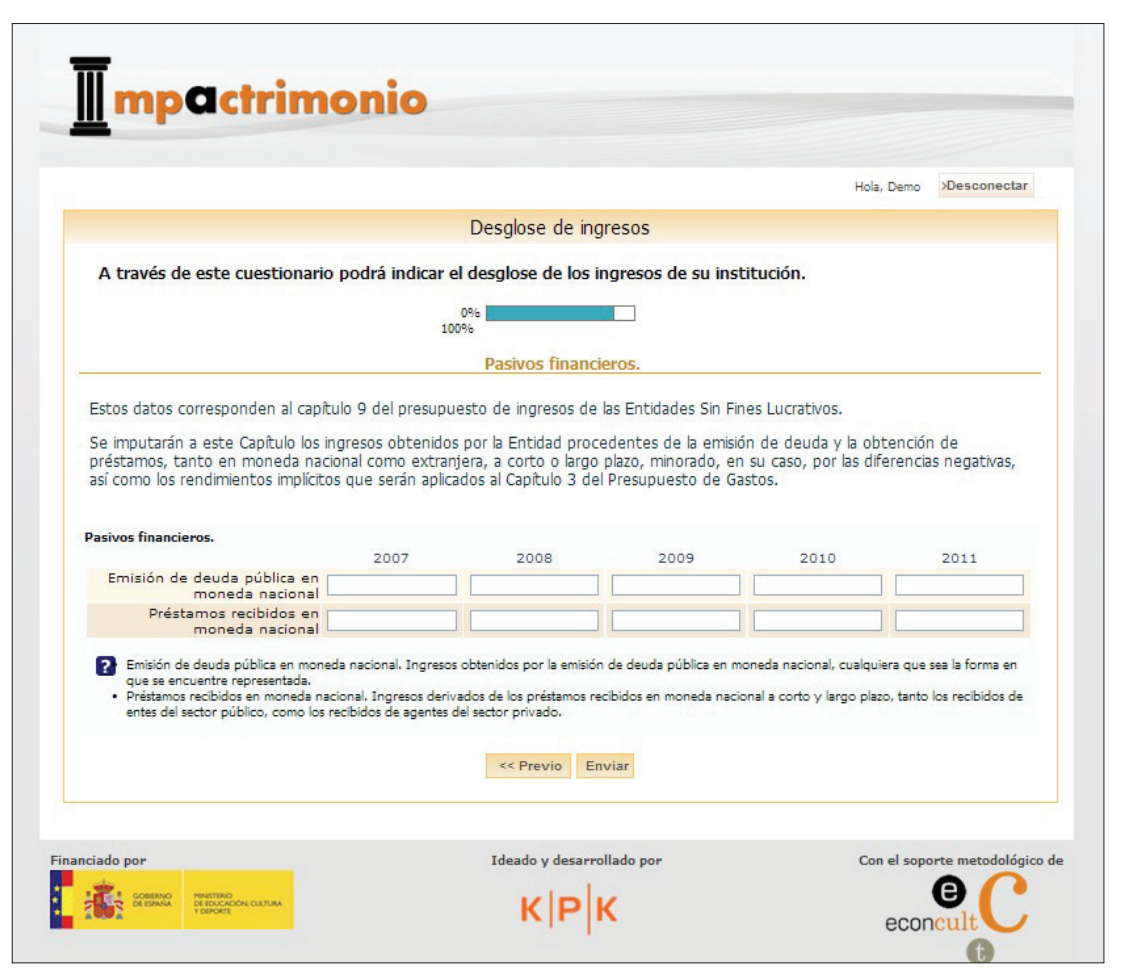

Figura 4. Cuestionario. Desglose de ingresos 
Su valor radica en dos aspectos: la creciente relevancia de la planificación y gestión cultural para el desarrollo socioeconómico, y las carencias existentes en materia de planificación y gestión cultural, especialmente en instrumentos de evaluación, imprescindibles para generar evidencias e incidir sobre las políticas.

El impacto total de una actividad cultural se obtiene de la suma de los efectos directos, indirectos e inducidos

Es un buen ejemplo de cómo las tecnologías de la información facilitan a los usuarios -de una manera sencilla, ágil y didáctica- la identificación, acceso, tratamiento y desarrollo de bases de datos y fuentes de información, generando informes útiles tanto para los gestores culturales como para todos aquellos implicados en los procesos de toma de decisiones y las políticas públicas relacionadas.

Para concluir se remarca que el modelo Impactrimonio es plenamente exportable, ya que puede adaptarse a otros contextos territoriales siempre que se disponga de las TIO y de los datos de los presupuestos públicos correspondientes.

\section{Agradecimientos}

El proyecto Impactrimonio ha sido financiado por la Subdirección General de Patrimonio Artístico del Ministerio de Educación, Cultura y Deporte de España en el marco del Plan de ayudas a empresas privadas para proyectos de puesta en valor, promoción, difusión y protección de bienes declarados patrimonio cultural; la Agencia Española de Cooperación Internacional al Desarrollo (registro 11-CAP2-1480) y el Ministerio de Economía y Competitividad (Plan Nacional de I+D+i 2008-2001, proyecto CSO2012-39373-C04-3).

\section{Nota}

1. La teoría convencional de la economía elabora sus modelos bajo los supuestos de información perfecta; es decir, todos los agentes tienen el nivel máximo posible de información de manera que en las transacciones todo el mundo es capaz de calcular la utilidad que le proporcionará un determinado bien o servicio. Sin embargo, todos somos conscientes de que en la realidad los agentes tienen niveles distintos de información (el ejemplo típico es la asimetría de información entre el vendedor de coches usados y el comprador), por lo que existe un desarrollo teórico de la "microeconomía", que se denomina "Economía de la información" que precisamente trata de analizar cómo cambian los equilibrios si consideramos que los agentes tienen distintos niveles de información. El artículo de Stigliz de 2000, precisamente es un "survey" donde se recogen las aportaciones más significativas de esta teoría. Los costos de información se refieren a los costes de obtener toda la información relevante para participar en un intercambio. Estos costes de información explican por ejemplo la aparición de "agencias de calificación" de la deuda.

\section{Bibliografía}

Comisión Europea (2010). Libro verde sobre el potencial de las industrias culturales y creativas. Bruselas: Comisión Europea.

http://europa.eu/legislation_summaries/culture/cu0006_ es.htm

Devesa, María (2006). El impacto económico de los festivales culturales. Madrid: Datautor. ISBN: 9788480487146

Herrero-Prieto, Luis-César (2002). "La economía de la cultura en España: una disciplina incipiente". Revista asturiana de economía, v. 23, pp. 147-175. http://giec.blogs.uva.es/files/2012/02/rae2002.pdf

Herrero-Prieto, Luis-César; Sanz, José-Ángel; Devesa, María; Bedate, Ana; Del-Barrio, María-José (2006). “The economic impact of cultural events: a case-study of Salamanca 2002, European Capital of Culture". European urban and regional studies, v. 13, n. 1, pp. 41-57.

KEA, European Affairs (2009). The economy of culture in Europe. Bruselas: European Commission Directorate General for Education and Culture.

http://ec.europa.eu/culture/key-documents/doc873_ en.htm

Pulido, Antonio; Fontela, Emilio (1993). Análisis input-output. Modelos, datos y aplicaciones. Madrid: Pirámide. ISBN: 9788436807561

Rausell-Köster, Pau; Abeledo-Sanchis, Raúl; Carrasco-Arroyo, Salvador; Martínez-Tormo, José (2007). Cultura: estrategia para el desarrollo local. Madrid: Agencia Española de Cooperación Internacional al Desarrollo. ISBN: 978848347 0329

http://www.cervantesvirtual.com/obra/cultura-estrategiapara-el-desarrollo-local--0

Rausell-Köster, Pau; Abeledo-Sanchis, Raúl et al. (2012). La cultura como factor de innovación económica y social. Proyecto Sostenuto, cofinanciado por el Programa Interreg IV B Med de la Unión Europea.

http://www.uv.es/raboixdo/references/2012/12012.pdf

Seeman, Bruce. (2003). "Economic impact of arts". En Towse, Ruth (ed.) A handbook of cultural economics. Cheltenham: Edward Elgar, pp. 224-231. ISBN: 1840643382

Shapiro, Carl; Varian, Hal R. (1999). Information rules: a strategic guide to the New York economy. Harvard Business Press. ISBN: 9780875848631

Stigliz, Joseph E. (2000). "The contributions of the economics of information to twentieth century economics". The quaterly journal of economics, v. 115, n. 4, pp. 1441-1478. http://dx.doi.org/10.1162/003355300555015 http://ricardo.ecn.wfu.edu/ cottrell/papers/stiglitz.pdf

UCLG (2004). Agenda 21 for culture. United cities and local governments - Committee on culture. http://agenda21culture.net

Unctad (2010). Creative economy report 2010. Ginebra: Unctad. ISBN: 9780981661902

http://unctad.org/es/Docs/ditctab20103_en.pdf 